\title{
VISUALIZATION AND QUANTIFICATION OF Dentin STRucture Using Confocal Laser SCANNing Microscopy
}

\author{
Yuichi Kimura, ${ }^{\dagger}$ Petra Wilder-Smith, ${ }^{\dagger}$ Tatiana B. Krasieva, ${ }^{*}$ Anna Marie A. Arrastia- \\ Jitosho, ${ }^{\dagger}$ Lih-Huei L. Liaw, ${ }^{*}$ and Koukichi Matsumoto ${ }^{\dagger}$ \\ ${ }^{\dagger}$ Department of Endodontics, Faculty of Dentistry, Showa University, 2-1-1, Kitasenzoku, Ohta-ku, \\ Tokyo 145, Japan; ${ }^{\star}$ Beckman Laser Institute and Medical Clinic, University of California, \\ Irvine, 1002 Health Sciences Road East, Irvine, California 92715, U.S.A. \\ (Paper JBO-115 received Oct. 15, 1996; revised manuscript received Apr. 21, 1997; accepted for publication May 7, 1997. )
}

\begin{abstract}
Dentin was visualized using a new fluorescence technique and confocal laser scanning microscopy. Thirty extracted human teeth showing no clinical signs of caries were investigated. All teeth were horizontally sectioned to approximately $200 \mu \mathrm{m}$ thickness and sections were subjected to different pretreatment conditions as follows: vacuum only, ultrasonication only, sodium hypochlorite only, sodium hypochlorite and vacuum, sodium hypochlorite and ultrasonication, and a combination of sodium hypochlorite, vacuum, and ultrasonication. Some samples were left untreated to serve as control. Following pretreatment, rhodamine 123 fluo-

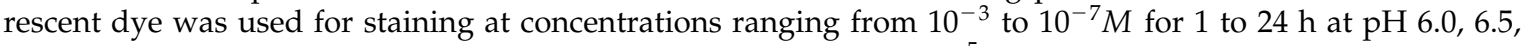
or 7.4. Optical staining occurred at $\mathrm{pH} 7.4$ and concentrations $\geqslant 10^{-5} \mathrm{M}$ over $3 \mathrm{~h}$ or longer. Surface images obtained using confocal laser scanning microscopy were similar to those observed by scanning electron microscopy without the need for sample-altering conventional scanning electron microscope preparation techniques. Subsurface imaging to a depth of approximately $60 \mu \mathrm{m}$ was achieved using confocal laser microscope techniques. This fluorescence technique offers a useful new alternative for visualization and quantification of dentin. () 1997 Society of Photo-Optical Instrumentation Engineers. [S1083-3668(97)00303-1]
\end{abstract}

Keywords dentin tubules, dental, imaging, fluorescent dye, tooth.

\section{INTRODUCTION}

Since the development of a scanning confocal optical system for the microscope in 1957, this device has been extensively modified and applied in many fields, including medicine. ${ }^{1}$ Scanning confocal microscopes offer improved rejection of out-of-focus light and greater resolution than conventional imaging. ${ }^{2}$ Today this form of microscopy represents a well-recognized technique in the fields of biological and materials science. In the dental field, scanning confocal microscopy has been mainly used for assessment of dental operative procedures and restorations, $^{3-6}$ study of the tooth/restoration interface, ${ }^{7}$ evaluation of enamel cutting interactions, ${ }^{8,9}$ visualization of enamel surface changes, ${ }^{10}$ and clinical dental research. ${ }^{11}$ However, this technique has not been used for visualization and quantification of dentin.

Confocal laser scanning microscope (CLSM) systems can scan a laser beam over stationary samples. ${ }^{12}$ Surface images obtained using the confocal microscope are similar in character to those provided by the scanning electron microscope (SEM), but subsurface imaging is also possible. Fur-

Address all correspondence to Petra Wilder-Smith. Tel: (714) 824-4713; Fax: (714) 824-8413; E-mail: pwsmith@bli.uci.edu thermore, confocal microscope images can be reconstructed three dimensionally. ${ }^{12,13}$ Conventional dehydration and gold-coating methods utilized for SEM have several disadvantages compared with sample preparation for CLSM: dehydration and heating can damage specimens and cause a variety of artifacts, such as cracking or "bubbling." Tooth samples can be visualized using the confocal laser scanning microscope either by lightly metal coating the surface or by using fluorescence techniques.

Two basic types of confocal microscopy exist: real-time direct view tandem scanning microscopy (TSM) and CLSM. ${ }^{12}$ The high frame speed of the TSM allows real-time examination of teeth in vivo. ${ }^{14,15}$ In contrast, all CLSMs rely on powerful image-processing devices and frame stocks for information displays. The acquisition of perfectly registered, serial optical sections by this technique provides an excellent means for the three-dimensional reconstruction and quantification of objects.

The purpose of this study was to develop a technique for using CLSM to image and study dentin. In addition to identifying appropriate dyes and fluorescence staining techniques, imaging depth and resolution were investigated. The results were 
Table 1 Overview of pretreatment conditions.

\begin{tabular}{lcccccc}
\hline Sample & $\begin{array}{c}\text { Sodium } \\
\text { hypochlorite }\end{array}$ & Vacuum & Ultrasonication & $\mathrm{pH}$ & $\begin{array}{c}\text { Dye } \\
\text { concentration } \\
(\mathrm{M})\end{array}$ & $\begin{array}{c}\text { Dye } \\
\text { exposure } \\
\text { (h) }\end{array}$ \\
\hline 1 & No & No & No & 7.4 & $10^{-5}$ & 3 \\
2 & No & Yes & No & 7.4 & $10^{-5}$ & 3 \\
3 & No & No & Yes & 7.4 & $10^{-5}$ & 3 \\
4 & Yes & No & No & 7.4 & $10^{-5}$ & 3 \\
5 & Yes & Yes & No & 7.4 & $10^{-5}$ & 3 \\
6 & Yes & No & Yes & 7.4 & $10^{-5}$ & 3 \\
7 & Yes & Yes & Yes & 7.4 & $10^{-5}$ & 3 \\
\hline
\end{tabular}

compared with images from conventional SEM techniques to contrast the levels of information obtained and to assess any structural effects of the staining techniques developed in this study.

\section{MATERIAls AND Methods}

\subsection{SAMPLE PREPARATION}

Thirty extracted human teeth showing no clinical signs of caries, stored in demineralized water with $0.01 \%(\mathrm{w} / \mathrm{v})$ thymol were horizontally sectioned into thin slices (approximately $200 \mu \mathrm{m}$ thickness) using a low-speed saw with coolant (Isomet, Buehler, Illinois).

\subsection{PRETREATMENT STUDY}

To determine optimal pretreatment conditions and procedures, we undertook the following preliminary investigations. Samples were treated as described in Table 1; sodium hypochlorite $(\mathrm{NaOCl})$ (5.25\% by wt, Darrow Co., California) application, vacuum, and/or ultrasonication treatments for $1 \mathrm{~h}$ were variable. After pretreatment, samples were subjected to staining procedures. Rhodamine 123 (Eastman Kodak Co., New York) with an absorption peak at $511 \mathrm{~nm}$ was used as the fluorescent dye because this absorption peak corresponds most closely to the excitation wavelength of $488 \mathrm{~nm}$ used in our confocal laser microscope. Staining was performed at the concentration of $10^{-5} \mathrm{M}$ for $3 \mathrm{~h}$. After staining, samples were rinsed 2 to 3 times with demineralized water for $5 \mathrm{~min}$. Sections were fixed to glass slides with cyanoacrylate glue and observed through the CLSM. Later, the same samples were prepared for conventional SEM procedures.

\subsection{STAINING STUDY}

To determine optimal fluorescence staining dyes and procedures, we undertook the following preliminary investigations. In untreated samples, rhodamine 123 concentrations of $10^{-3}, 10^{-4}$,
$10^{-5}, 10^{-6}$, and $10^{-7} \mathrm{M}$ were tested at $\mathrm{pH} 6.0,6.5$, or 7.4 and staining durations of $1,2,3,4$, or $24 \mathrm{~h}$. The buffers used included $50 \mathrm{mM}$ (2N-morpholino) ethanesulfonic acid (MES) at $\mathrm{pH}$ 6.0, $50 \mathrm{mM}$ piperazine- $N, N^{\prime}$-bis(2-ethanesulfonic acid) (PIPES) at $\mathrm{pH} 6.5$, and $10 \mathrm{mM}$ phosphate buffer saline (PBS) at $\mathrm{pH}$ 7.4. After staining, samples were rinsed 2 to 3 times with demineralized water for $5 \mathrm{~min}$. Sections were fixed on glass slides with cyanoacrylate glue, and surface staining intensity was determined by conventional fluorescence microscopy (Carl Zeiss, Oberkochen, Germany). The effects on staining of ultrasonication and vacuum application for $1 \mathrm{~h}$ were also evaluated.

\section{SCANNING ELECTRON MICROSCOPY}

Scanning electron microscopy was used to identify the structural effects of the different variables in the fluorescence staining techniques. The samples were dehydrated in a graded series of aqueous ethanol $(30,50,70,90$, and $100 \%$ ethanol) for $10 \mathrm{~min}$ at each concentration, mounted on stubs using colloidal silver liquid (Ted Pella, California) and gold coated on a PAC-1 Pelco advanced coater 9500 (Ted Pella). Micrographs of the dentin surface were taken on a Philips 515 (Mohawk, New Jersey) SEM. Some samples were manually split at 90 deg to the surface, then gold coated to allow visualization of subsurface structures.

\subsection{CONFOCAL LASER MICROSCOPE DEVICE}

Stained samples were examined using an LSM 410 inverted Zeiss laser scanning microscope (Carl Zeiss, Oberkochen, Germany). Stacks of thin optical sections were obtained for each sample. The objective lens used was the Plan-Neofluar $\times 100$ bright field, n.a.1.3, oil immersion (Carl Zeiss, Oberkochen, Germany). The laser wavelength of $488 \mathrm{~nm}$ was used for fluorescence excitation; emission was isolated with a long-pass 520-nm filter. The distance between optical sections was $2 \mu \mathrm{m}$ on 

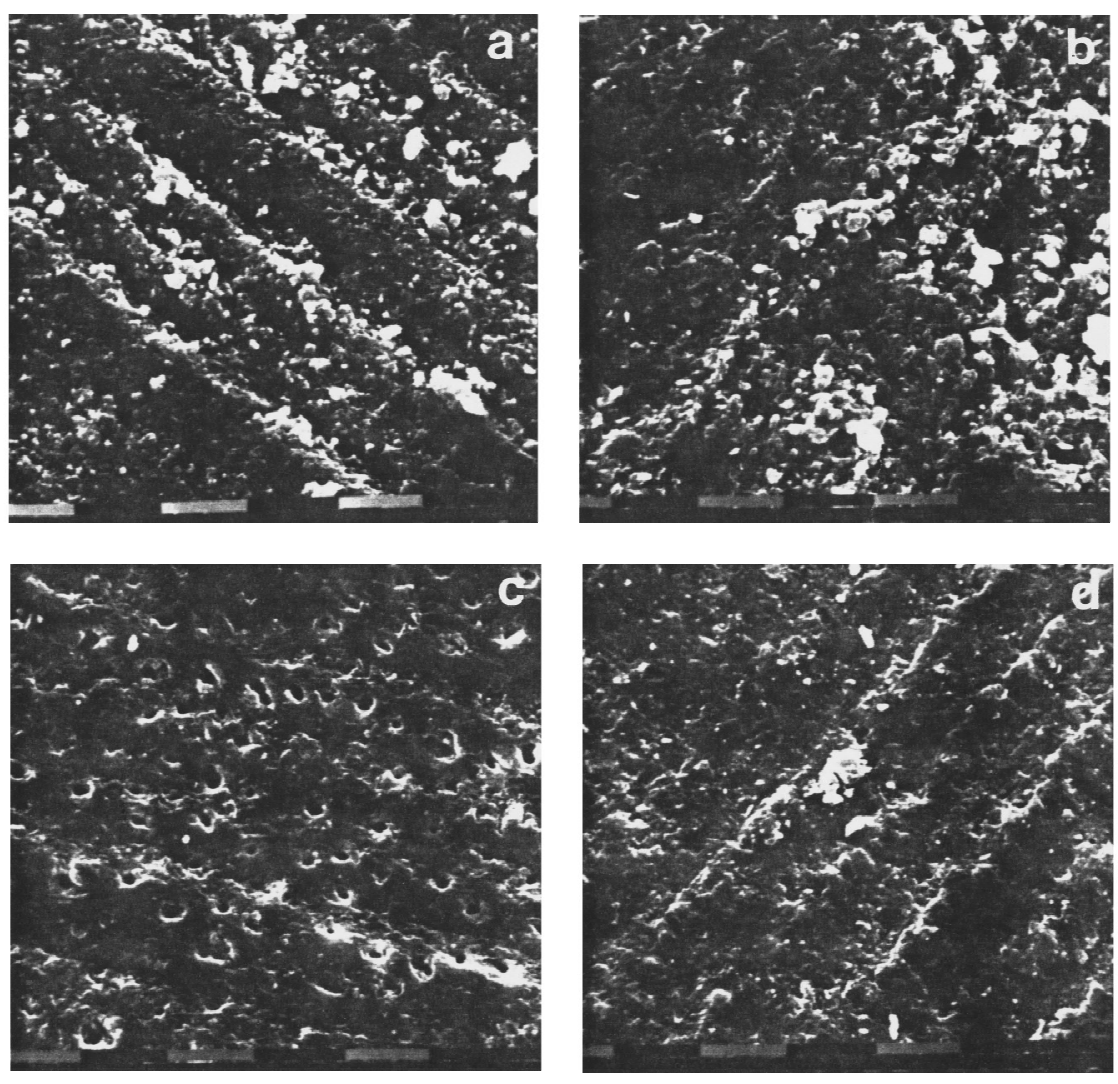

Fig. 1 SEM photographs: (a) no treatment, (b) vacuum treatment (for 1 h), (c) ultrasonication treatment (for 1 h), (d) sodium hypochlorite treatment (for $1 \mathrm{~h}$ ), (e) sodium hypochlorite and vacuum treatment (for $1 \mathrm{~h}$ ), (f) sodium hypochlorite and ultrasonication treatment (for $1 \mathrm{~h}$ ), and (g) sodium hypochlorite, vacuum, and ultrasonication treatment (for $1 \mathrm{~h})$. $\times 1010$. The scale shows $10 \mu \mathrm{m}$.

the $z$ axis. Overall depth of acquisition ranged from approximately 20 to $60 \mu \mathrm{m}$, depending on depth of penetration of rhodamine 123 into the sample. The information obtained was stored on a 1-GByte optical disk (Panasonic) and three-dimensional images were generated from stacks of stored images using original LSM 410 software.

\section{RESULTS}

\subsection{PRETREATMENT STUDY}

Figures 1(a) through $1(\mathrm{~g})$ show SEM photographs of samples after various pretreatments. Figures 2(a) through 2(g) show CLSM images of the same samples as in Figure 1. In Figures 1(a) and 2(a) (untreated samples), SEM showed surface debris and ridges from sample preparation with the saw. With CLSM, the surface image obtained was difficult to capture in focus, due to surface unevenness and focusing limitations. However, saw marks and surface debris are again visible. Subsurface images at 2 $\mu \mathrm{m}$ and deeper, showing dentin tubules, are much clearer than surface images and appear in focus.

In Figures 1(b) and 2(b) (vacuum treatment samples), SEM showed surface debris and ridges from sample preparation with the saw. With CLSM, the surface image was again difficult to capture in focus, due to surface unevenness and focusing limitations. However, saw marks and surface debris are again visible, and subsurface images at $2 \mu \mathrm{m}$ and deeper, showing dentin tubules, are much clearer and appear in focus.

In Figures 1(c) and 2(c) (ultrasonication treatment samples), surface images obtained by SEM and CLSM are comparable. No surface debris was evident. Subsurface imaging was successful to a depth of $18 \mu \mathrm{m}$.

Surface images obtained by SEM and CLSM are very similar for Figures 1(d) and 2(d) (sodium hypochlorite treatment samples). Minimal surface debris was observed. Subsurface imaging was successful to a depth of $30 \mu \mathrm{m}$. Tubule diameters appeared larger than in previous samples, with tubular walls showing marked fluorescence.

In Figures 1(e) and 2(e) (sodium hypochlorite and vacuum treatment samples), surface images obtained by SEM and CLSM are comparable. No surface debris was evident. Subsurface imaging was successful to a depth of $22 \mu \mathrm{m}$. Using CLSM, the surface image obtained was difficult to capture in focus, due to surface unevenness and focusing limitations.

In Figures 1(f) and 2(f) (sodium hypochlorite and ultrasonication treatment samples), surface images 

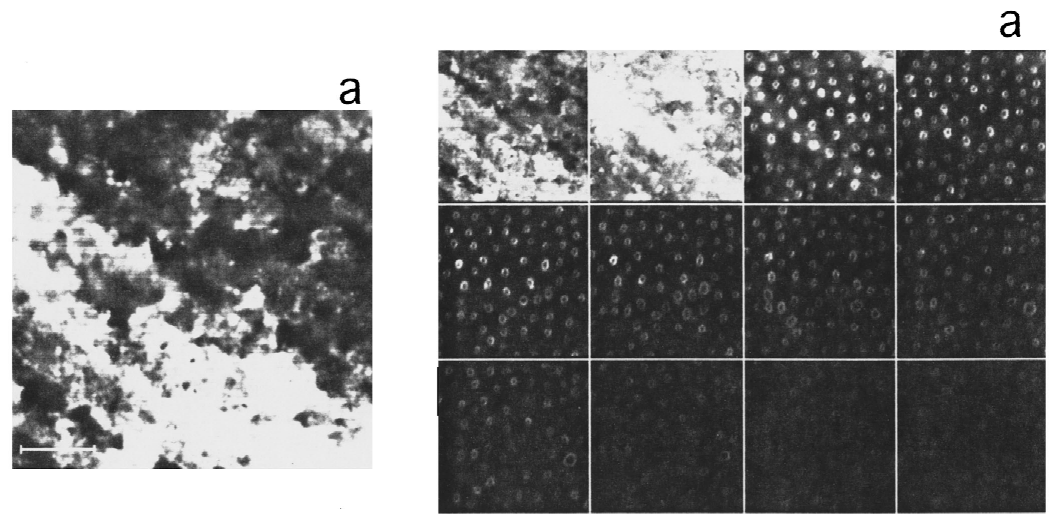

b

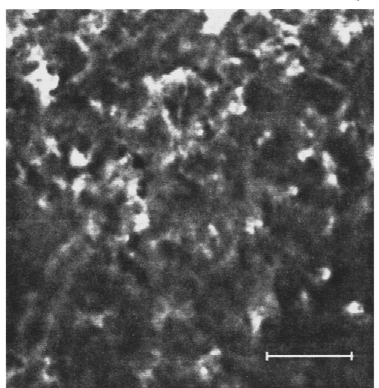

b
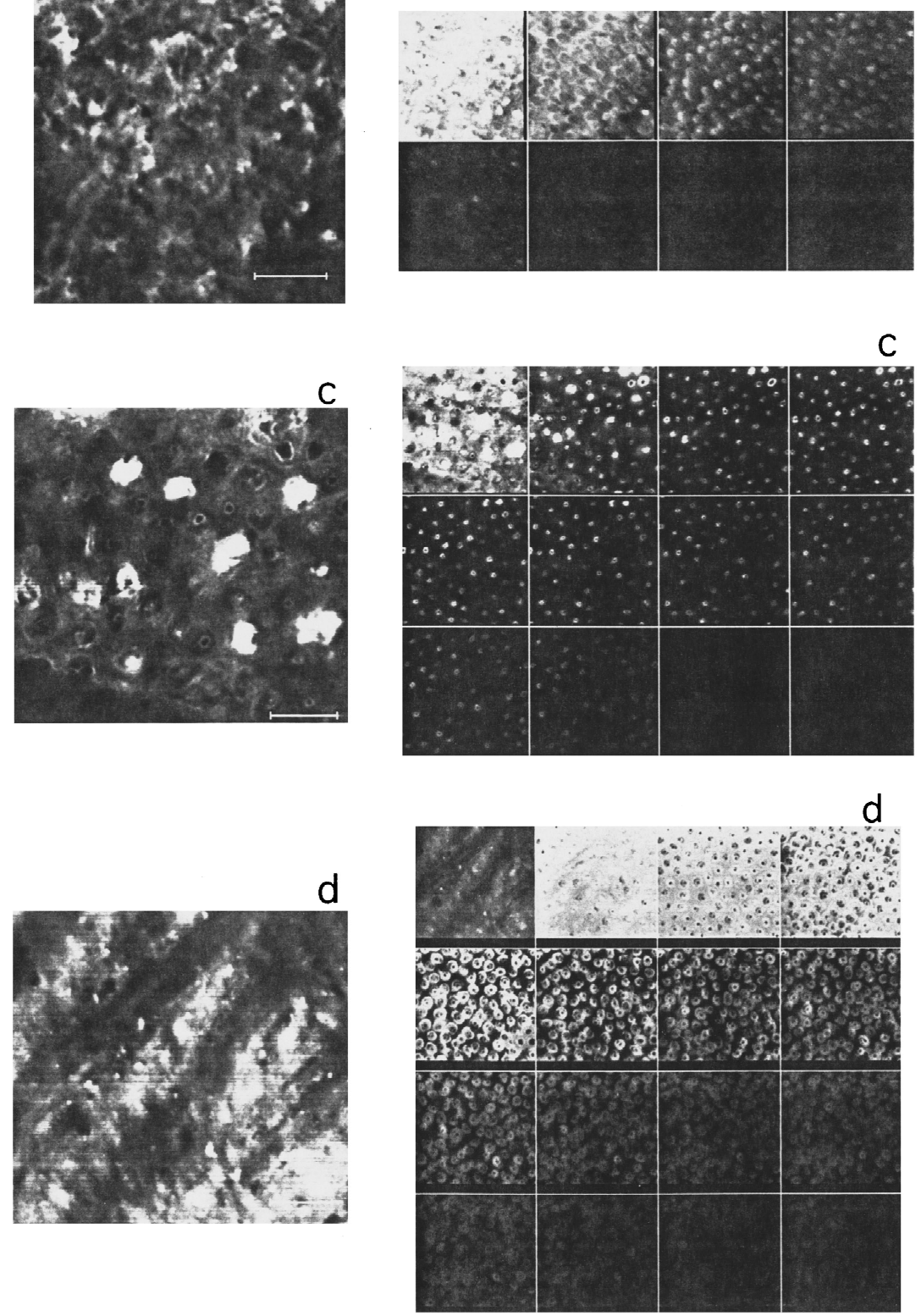

Fig. 2 CLSM images on the same samples as in Figure 1: (a) no treatment, (b) vacuum treatment (for $1 \mathrm{~h}$ ), (c) ultrasonication treatment (for 1 h), (d) sodium hypochlorite treatment (for 1 h), (e) sodium hypochlorite and vacuum treatment (for 1 h), (f) sodium hypochlorite and ultrasonication treatment (for $1 \mathrm{~h}$ ), and (g) sodium hypochlorite, vacuum, and ultrasonication treatment (for $1 \mathrm{~h}$ ). Each figure is a pair of surface view (left) and a series of $2-\mu \mathrm{m}$ sections (right). $\times 1000$. The scale shows $10 \mu \mathrm{m}$.

270 Journal of Biomedical Optics • July 1997 • Vol. 2 No. 3 

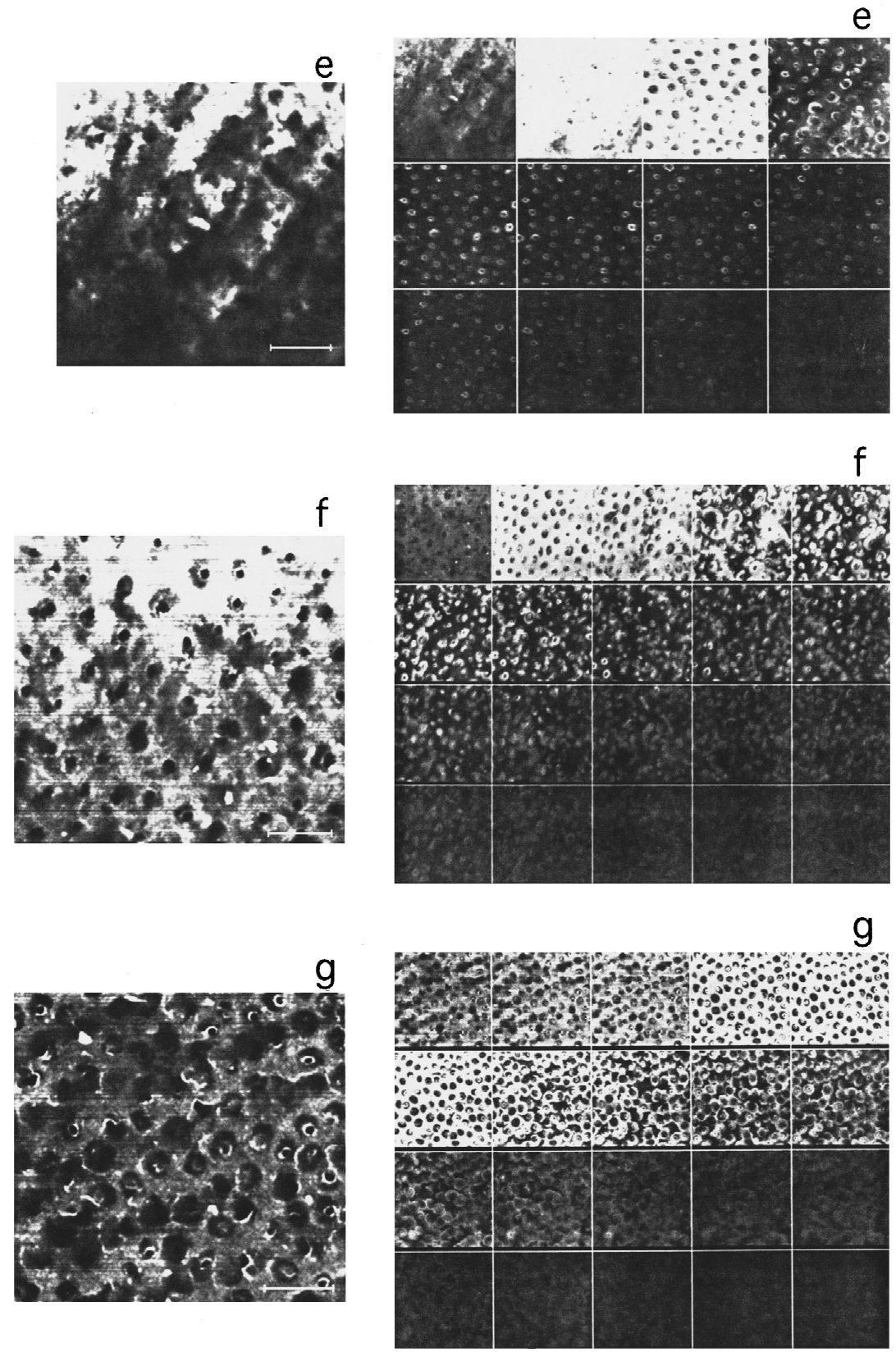

Fig. 2 (Continued).

obtained by SEM and CLSM are comparable. Subsurface imaging was successful to a depth of $38 \mu \mathrm{m}$, and no surface debris was observed. Tubule diameters appeared larger than in previous samples, with tubular walls showing marked fluorescence.

In Figures 1(g) and 2(g) (sodium hypochlorite, vacuum and ultrasonication treatment samples), surface images obtained by SEM and CLSM are similar. No surface debris was observed and subsurface imaging was successful to a depth of $38 \mu \mathrm{m}$. Tubule diameters appeared larger than in previous samples, with tubular walls showing marked fluorescence.
Especially in untreated samples, the smear layer caused by sample sectioning prevented a clear view of dentin tubular structure. Pretreatment with a combination of sodium hypochlorite, vacuum, and ultrasonication permitted visualization at the deepest level, $38 \mu \mathrm{m}$. The CLSM surface views of the sections were very similar to SEM images.

In addition, subsurface structures could be observed with CLSM due to the permeability of the smear layer to the fluorescence dye, rhodamine 123. Dye penetration and subsurface visualization were improved by using sodium hypochlorite and ultrasonication or a combination of sodium hypochlo- 

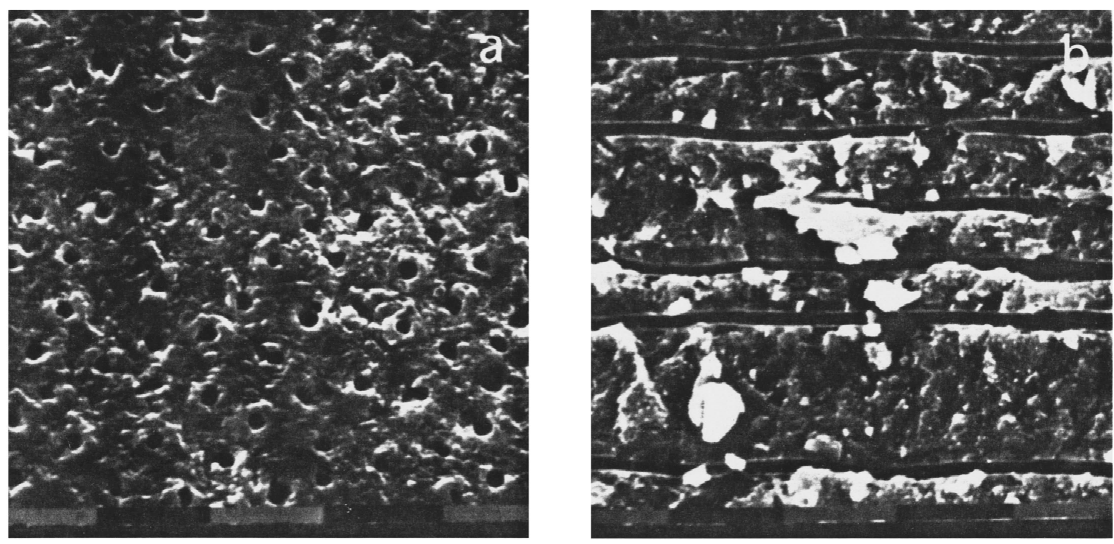

Fig. 3 SEM photographs: (a) surface view and (b) lateral view. Sample was pretreated with a combination of sodium hypochlorite, vacuum, and ultrasonication (for $1 \mathrm{~h}$ ). $\times 1010$. The scale shows $10 \mu \mathrm{m}$.

rite, vacuum, and ultrasonication. This methodology permitted visualization of samples to a depth of approximately 30 to $50 \mu \mathrm{m}$ after pretreatment, compared with control samples, where visualization was possible only to a depth of approximately $20 \mu \mathrm{m}$. In general, dye penetration was good after pretreatment, but intersample variability measured up to a factor of 3 .

\subsection{STAINING STUDY}

Rhodamine 123 concentrations ranging from $10^{-3}$ to $10^{-7} \mathrm{M}$ were evaluated at $\mathrm{pH} 7.4$ for $3 \mathrm{~h}$ exposure time. Concentration $\geqslant 10^{-5} \mathrm{M}$ resulted in goodquality images. Staining effectiveness at a $\mathrm{pH}$ of 6.0, 6.5 , or 7.4 was investigated. Rhodamine 123 staining was best at a $\mathrm{pH}$ of 7.4. Immersion times $\geqslant 3 \mathrm{~h}$ resulted in good images. Effects of ultrasonication and vacuum pretreatment were small, but these techniques reduced the required time for immersion.

\subsection{COMPARISON BETWEEN SEM AND CLSM}

Figure 3 shows SEM photographs. Figure 3(a) shows the surface view and Figure $3(\mathrm{~b})$ shows the lateral view. Figure 4 shows CLSM images in the same sample as in Figure 3. Figure 4(a) shows the surface view; Figure $4(\mathrm{~b})$ shows subsurface scans; Figure 4(c) shows the accumulated 3-D view (0 deg); and Figure 4(d) shows the accumulated 3-D lateral view $(90 \mathrm{deg})$. The images obtained by SEM or CLSM were very similar.

\section{DISCUSSION}

A limited range of applications for scanning confocal microscopy has been employed in the field of dentistry. Usually applications involve methods for assessing dental operative procedures and materials. ${ }^{3-11}$ By eliminating the need for problematic specimen preparation techniques, confocal microscopy is well suited to the observation of dental and material surfaces and to monitoring the effects of various agents and factors on their microstructure. In these earlier studies, however, TSM was mainly used, although the use of CLSM was also reported. ${ }^{12}$

Other dental applications of CLSM have been reported, ${ }^{10}$ but using metal-coated samples, rather than fluorescent staining techniques. In addition to its costly, demanding, and time-consuming nature, metal coating has the added disadvantage of usually requiring sample dehydration, a process that can easily damage, alter, or distort sample structure and produce multiple artifacts.

Compared with SEM, magnification provided by confocal microscopy and fluorescence techniques is low (maximum $\times 1000$ ), but within this range CLSM allows surface and subsurface visualization in three dimensions using a fluorescent dye. Dimensional quantification is easy and accurate: thus we were able to follow and measure the course and dimensions of dentin tubules easily using marker systems on the computer screen. The disadvantages of CLSM techniques include its lack of suitability for clinical investigations because relatively thin sample sections are needed. However, the highframe speed of the TSM enables real-time examination of teeth in vivo. ${ }^{14,15}$

Our investigations of fluorescence staining techniques for dentin showed that sodium hypochlorite application only did not enhance dye uptake, but was very effective together with ultrasonication in achieving good staining. Similar surface images were obtained using SEM and CLSM, but it was possible to also perform noninvasive subsurface imaging in dentin using CLSM. The presence of a smear layer reduced the effectiveness of fluorescence staining, but the smear layer was partially permeable to the fluorescent dye. Using the fluorescence techniques developed in this investigation, we were able to achieve good surface and subsurface images of dentin samples. Optimum staining conditions for visualization and quantification of dentinal structure were identified in this study. 

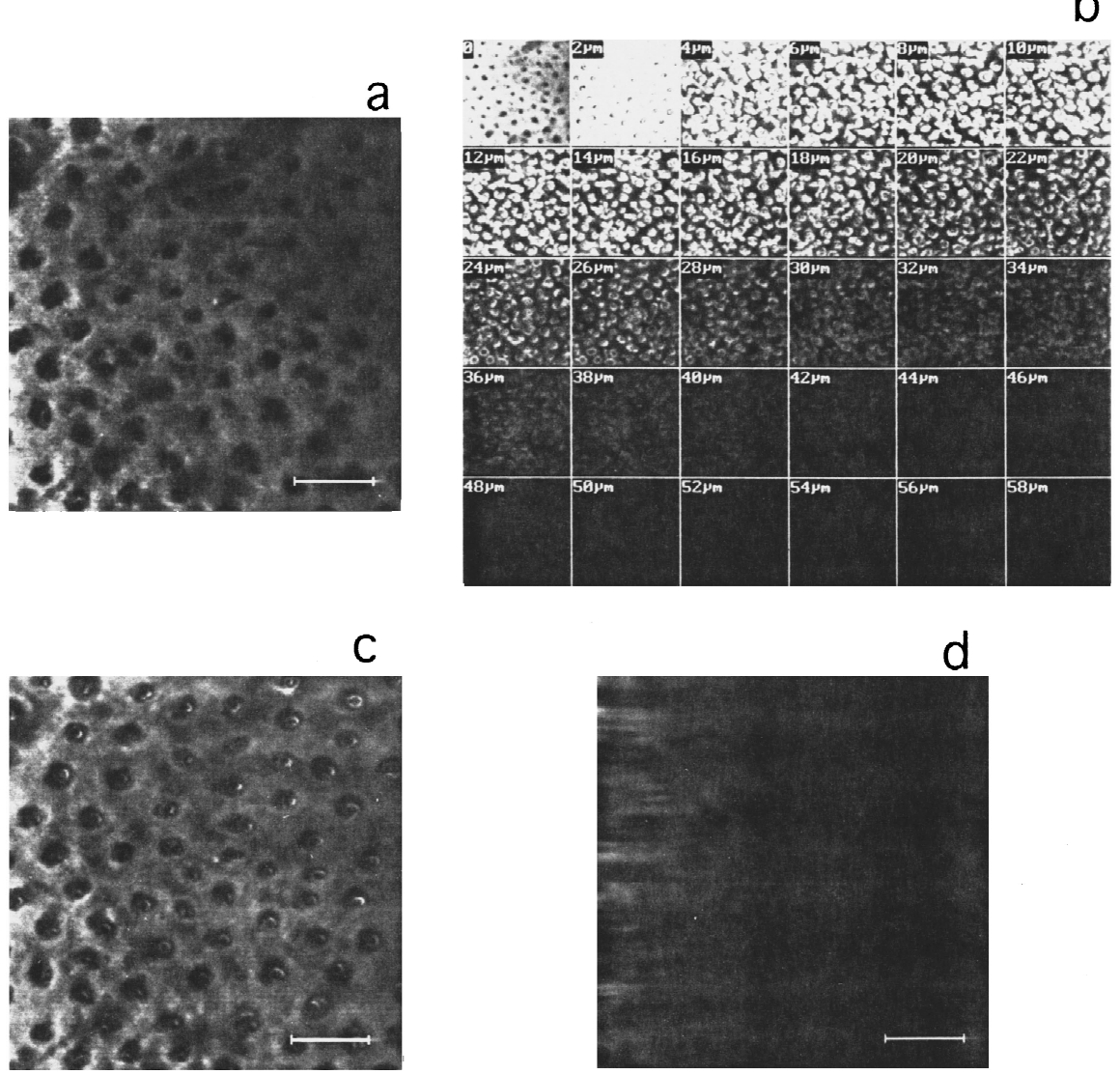

Fig. $4 \mathrm{CLSM}$ images of the same sample as in Figure 3: (a) surface view, (b) series of 2- $\mu \mathrm{m}$ sections, (c) accumulated 3-D view (0 deg), and (d) accumulated 3-D lateral view $(90 \mathrm{deg}) . \times 1000$. The scale shows $10 \mu \mathrm{m}$.

However, further improvements in subsurface visualization would be beneficial.

\section{Acknowledgments}

This study was supported by U.S. Department of Energy Grant DE903-91ER61227, Office of Naval Research Grant N00014-90-0-0029, and National Institutes of Health Grants RR01192 and CA-62203. It was made possible, in part, through access to the Laser Microbeam and Medical Program (LAMMP) and the Clinical Cancer Center Optical Biology Shared Resource at the University of California, Irvine. We would like to thank Dr. Michael W. Berns for his advice and support. We are also indebted to Mr. A. Nguyen and B. Tran for their assistance in this study.

\section{REFERENCES}

1. S. Inoue, "Foundations of confocal scanned imaging in light microscopy," in Handbook of Biological Confocal Microscopy, J. B. Pawley, ed., Chap. 1, pp. 1-14, Plenum Press, New York (1990).

2. J. G. White, W. B. Amos, and M. Fordham, "An evaluation of confocal versus conventional imaging of biological structures by fluorescence light microscopy," J. Cell Biol. 105, 41-48 (1987).
3. T. F. Watson, "A confocal optical microscope study of the morphology of the tooth/restoration interface using Scotchbond 2 dentin adhesive," J. Dent. Res. 68(6), 1124-1131 (1989).

4. T. F. Watson, "A confocal microscopic study of some factors affecting the adaptation of a light-cured glass ionomer to tooth tissue," J. Dent. Res. 69(8), 1531-1538 (1990).

5. T. F. Watson, R. W. Billington, and J. A. Williams, "The interfacial region of the tooth/glass ionomer restoration: a confocal optical microscope study," Am. J. Dent. 4(6), 303310 (1991).

6. T. F. Watson and D. M. de J. Wilmot, "A confocal microscopic evaluation of the interface between Syntac adhesive and tooth tissue," J. Dent. 20, 302-310 (1992).

7. M. Torabinejad, T. F. Watson, and T. R. Pitt Ford, "Sealing ability of a mineral trioxide aggregate when used as a root end filling material," J. Endodon. 19(12), 591-595 (1993).

8. T. F. Watson, "The application of real-time confocal microscopy to the study of high-speed dental-bur-tooth-cutting interactions," J. Microscop. 157(1), 51-60 (1990).

9. T. F. Watson, "Tandem-scanning microscopy of slow speed enamel cutting interactions," J. Dent. Res. 70(1), 44-49 (1991).

10. T. Kodaka, M. Kuroiwa, and M. Kobori, "Scanning laser microscopic surface profiles of human enamel and dentin after brushing with abrasive dentifrice in vitro," Scanning Microscop. 7, 245-254 (1993).

11. T. F. Watson, W. M. Petroll, H. D. Cavanagh, and J. V. Jester, "In vivo confocal microscope in clinical dental research: an initial appraisal," J. Dent. 20, 352-358 (1992).

12. T. F. Watson, "Applications of confocal scanning optical microscopy to dentistry," Br. Dent. J. 9, 287-291 (1991). 
13. K. Carlsson, P. E. Danielsson, R. Lenz, A. Liljeborg, L. Majlof, and N. Aslund, "Three-dimensional microscopy using a confocal laser scanning microscope," Opt. Lett. 10(2), 53-55 (1985).

14. K. C. New, W. M. Petroll, A. Boyde, L. Martin, P. Corcuff, J. L. Leveque, M. A. Lemp, H. D. Cavanagh, and J. V. Jester,
"In vivo imaging of human teeth and skin using real-time confocal microscopy," Scanning 13, 369-372 (1991).

15. T. F. Watson, "Applications of high-speed confocal imaging techniques in operative dentistry," Scanning 16, 168-173 (1994).

274 Journal of Biomedical Optics • July 1997 - Vol. 2 No. 3 